\title{
EVALUACIÓN DE LA GOBERNANZA DEL PROGRAMA RED DE OPORTUNIDADES DE PANAMÁ: LOGROS ALCANZADOS Y RETOS PENDIENTES
}

\author{
MARTINA ISABEL HIM CAMAÑO \\ Facultad de Economía. Campus de Veraguas. UNIVERSIDAD DE PANAMÁ, PANAMÁ \\ e-mail: m_himca@hotmail.com \\ BELÉN MIRANDA ESCOLAR \\ Departamento de Economía Aplicada. UNIVERSIDAD DE VALLADOLID, ESPAÑA \\ e-mail: belen@eco.uva.es \\ HELENA CORRALES HERRERO \\ Departamento de Economía Aplicada. UNIVERSIDAD DE VALLADOLID, ESPAÑA \\ e-mail: helena@eaee.uva.es \\ OLGA OGANDO CANABAL \\ Departamento de Economía Aplicada. UNIVERSIDAD DE VALLADOLID, ESPAÑA \\ e-mail: ogando@eco.uva.es
}

\section{RESUMEN}

Evaluar una política pública supone adoptar una postura abierta en materia de metodologías y técnicas (cuantitativas y cualitativas) a utilizar. Mientras las técnicas cuantitativas responden a la pregunta cuánto, las de carácter cualitativo intentan averiguar cómo y por qué unas intervenciones funcionan y otras no. El objetivo de este trabajo es evaluar, a partir de técnicas cualitativas, la gobernanza del programa Red de Oportunidades de Panamá, dado que el marco institucional se ha convertido en un aspecto decisivo para el éxito de las políticas públicas. Se han identificado errores de exclusión e inclusión en el proceso de selección de los beneficiarios del programa y otras deficiencias en la gobernanza, tales como la necesidad de contar con protocolos más eficaces al objeto de garantizar el seguimiento de las corresponsabilidades en educación.

Palabras clave: evaluación, gobernanza, transferencias monetarias condicionadas, Red de Oportunidades, Panamá

\section{Evaluation of governance of the Panama's Red de Oportunidades programme: achievements and pending challenges}

\begin{abstract}
Public policy evaluation implies adopting an open attitude to methodologies and techniques (quantitative and qualitative). While quantitative techniques answer the question how much, those of a qualitative nature try to find out how and why some interventions work and others do not. The objective of this paper is to evaluate, using qualitative techniques, the governance of Panama's Red de Oportunidades programme, given that the institutional framework has become a decisive aspect for the success of public policies. Exclusion and inclusion errors in the identification of programme beneficiaries and other deficiencies in governance have been detected, such as the need to stablish protocols more effective in order to guarantee the monitoring of co-responsibilities in education.
\end{abstract}

Keywords: evaluation, governance, conditional cash transfers, Red de Oportunidades, Panama.

Clasificación JEL: I28, I38 


\section{INTRODUCCIÓN}

Evaluar un programa o una política pública supone adoptar una visión abierta en materia de metodologías y técnicas (cuantitativas y cualitativas) a utilizar, así como asumir diferentes corrientes de pensamiento dentro de las ciencias sociales y de la teoría de la administración pública. No se trata únicamente de la aplicación de una metodología o una técnica; es, también, un enfoque a través del cual el evaluador se acerca al objeto de evaluación con su propia posición ante la realidad, con su visión sobre el objeto a estudiar, con paradigmas emergentes y dominantes y con el contexto en el que se desenvuelve la tarea evaluativa.

Este trabajo se centra básicamente en dos tipos de evaluación: formativa, enfocada a la implementación y ejecución del programa buscando la mejora y el cambio internos; y participativa, que pretende el fortalecimiento institucional a partir de metodologías de desarrollo organizacional. Ambas se aplican al programa Red de Oportunidades de Panamá, prestando especial atención a los factores que pueden dificultar las tareas de seguimiento y evaluación. En concreto: factores culturales, que tienen que ver con la actitud, el comportamiento y la mentalidad de los actores; factores metodológicos, asociados a los requerimientos y exigencias que impone el método científico y que interfieren con el modus operandi y la propia lógica de las políticas públicas; y factores estructurales, relacionados con la arquitectura y el funcionamiento de las organizaciones e individuos involucrados en el programa.

Las instituciones desempeñan un papel clave en la forma en que las sociedades distribuyen los beneficios y los costes de las políticas públicas que se ponen en marcha, siendo imprescindible contar con normas sólidas que afecten a la calidad de la información, a la transparencia en la gestión y al ejercicio de un buen gobierno. Sin estados eficaces que trabajen junto a ciudadanos activos y comprometidos hay pocas posibilidades de que tenga lugar el cambio que se necesita. Y es aquí, ayudando a las instituciones a iniciar ese cambio, donde la evaluación de la gobernanza de las políticas públicas tiene sentido. Porque tal vez los mayores beneficios de la evaluación no sean los resultados directos que genera en términos de lo que pondría de manifiesto una evaluación de impacto, sino la dinámica de aprendizaje que puede estimular entre los responsables políticos y los gestores públicos, así como en los demás actores involucrados, las mejoras en gobernanza o los cambios institucionales que se pueden derivar de ella (Lehtonen, 2005, 2006; Grin y Loeber, 2007; Betts y Wedgwood, 2011; International NGO Training and Research Centre, 2013). Bajo esta perspectiva la evaluación es un proceso más que una mera técnica.

Una evaluación de gobernanza requiere la incorporación en el análisis del contexto político y administrativo en el que se desarrolla el programa. Compromete a los actores, a los procesos, a las estructuras y a las instituciones responsables, e intenta averiguar quién hace qué y cómo lo hace. Cuando se sabe el porqué, existe una base sólida sobre la que actuar para poder mejorar la intervención. Para ello se aconseja mirar en el interior de la caja negra, donde se encuentra todo lo que ocurre en el programa; acciones, decisiones, recursos, personas e interacciones. Se espera que la combinación de todos estos factores produzca los resultados previstos. Realmente un programa es esto, ya que los resultados son las salidas que incluso pueden considerarse una variable exógena porque es lo que trasciende de los límites de la intervención (Legero, 2011).

Frente a la evaluación de la gobernanza se encuentra la gobernanza de la evaluación, es decir, el conjunto de mecanismos y procesos utilizados para gestionar la evaluación, encaminados a asegurar su efectividad y a hacer un uso completo de la información generada. Requiere tres elementos: desarrollar directrices para la evaluación, asegurar imparcialidad e independencia, y vincular los resultados de la evaluación a futuras intervenciones.

Un sistema de gobernanza bien diseñado garantiza que las decisiones se adoptan de manera coherente y clara, permite la participación activa de los stakeholders y fomenta la confianza de la sociedad en la forma en que se gastan los fondos públicos. Un elemento determinante en la gobernanza de un programa o de una política pública lo constituye el hecho de disponer de un buen sistema de seguimiento y evaluación y de una batería de indicadores adecuados para tal finalidad. Para ello, es imprescindible contar con estructuras especializadas que tengan capacidad y autonomía de gestión (Barret y Kidd, 2015) y que se encarguen de recabar la información necesaria. Un sistema de 
seguimiento y evaluación para un programa de las características de la Red de Oportunidades debería tener como mínimo: un registro de inscripción de beneficiarios; un sistema de supervisión y control de las condicionalidades; un mecanismo de control de pagos y de sanciones; un sistema de alerta que identifique a los beneficiarios que deben salir del programa; y una batería de indicadores SMART (Specific, Measurable, Achievable, Robustness and Timely) y CLEAR (European Commission, 2012, 2014).

El objetivo de este artículo es realizar una evaluación cualitativa del programa Red de Oportunidades de Panamá, prestando especial atención a la participación de los actores implicados, a los mecanismos de coordinación interinstitucionales y a la aplicación de los principales parámetros (focalización y condicionalidad en educación). Concretamente, se ha trabajado con datos primarios recabados de diferentes actores involucrados en la gestión y control del programa, lo que permite una mejor aproximación al contemplarlo desde distintas perspectivas. El artículo se estructura en cinco epigrafes. Tras la introducción, se describen las características más importantes del programa Red de Oportunidades pasando, a continuación, a explicitar la metodología utilizada y los datos. Finalmente, se presentan los principales resultados de la investigación y las conclusiones más relevantes.

\section{EL PROGRAMA RED DE OPORTUNIDADES DE PANAMÁ}

En 2006 entra en vigor el programa Red de Oportunidades de Panamá con el propósito de aliviar de manera directa, temporal e integral las necesidades inmediatas de los hogares en situación de pobreza extrema. El programa establece un sistema de incentivos (transferencias monetarias condicionadas, TMC) que se conceden a estos hogares con el fin de que inviertan en el capital humano de sus hijos y hagan un mayor uso de los servicios de educación y salud materno-infantil. A cambio, los beneficiarios de la Red asumen el compromiso de mantener la escolaridad de los niños y de participar en diversos programas de capacitación para el desarrollo de habilidades que permitan la obtención de ingresos.

Inicialmente el programa se financiaba con fondos provenientes de partidas discrecionales de la Presidencia de la República de Panamá, pero, a partir de 2009, contribuyen de manera conjunta el Banco Mundial (BM) y el Banco Interamericano de Desarrollo (BID), en el marco de un convenio de cooperación. La aportación para el conjunto del período 2009-2014 ascendía a un total de 46,94 millones de dólares, 20,17 millones de financiación por parte del BID y 24,0 millones aportados por el $\mathrm{BM}$, no destinados al pago de la transferencia sino a financiar un conjunto de intervenciones complementarias. Los 2,77 millones de dólares restantes y el Fondo Especial para la Pobreza Extrema, cuya fuente de ingresos procede de los recursos que genera el Canal de Panamá, con una dotación total de 161 millones', se destinaría al pago de las transferencias monetarias. Desde el año 2010, el programa Red de Oportunidades se inserta dentro del Plan Estratégico de Gobierno de Panamá.

\subsection{Los parámetros del programa}

Los parámetros constituyen la base que define la estructura organizacional y los procedimientos a seguir hasta que la transferencia llega al beneficiario final y se comprueba que se cumple con las condiciones establecidas. Los principales son: (i) los criterios de elegibilidad (focalización); (ii) la estructura de beneficios; (iii) la condicionalidad; (iv) las sanciones; y (v) el sistema de recertificación y salida (Tabla 1).

\footnotetext{
- Montante inicialmente presupuestado en el Plan Estratégico de Gobierno de Panamá 2010-2014.
} 
Tabla 1

Principales parámetros del componente de transferencias monetarias condicionadas del programa Red de Oportunidades

Focalización

\begin{tabular}{|c|c|c|c|c|c|}
\hline \multicolumn{2}{|l|}{ Clase } & Indicador & \multicolumn{3}{|c|}{ Sistema de información } \\
\hline \multicolumn{2}{|l|}{ Geográfica } & $\begin{array}{l}\text { Índice Compuesto de Marginalidad } \\
\text { (ICM) }\end{array}$ & \multicolumn{3}{|c|}{$\begin{array}{l}\text { Sistema de Información Gerencial del Programa } \\
\text { (SIG) }\end{array}$} \\
\hline \multicolumn{2}{|c|}{$\begin{array}{l}\text { Por hogar. } \\
\text { Comprobación de medios }\end{array}$} & Proxy means test (PMT) & \multicolumn{3}{|c|}{ Encuesta de Vulnerabilidad Social (EVS) } \\
\hline \multicolumn{6}{|c|}{ Estructura de beneficios } \\
\hline \multirow{2}{*}{$\begin{array}{l}\text { Beneficiarios a los } \\
\text { que está dirigido }\end{array}$} & \multirow{2}{*}{\multicolumn{2}{|c|}{ Transferencias }} & & \multicolumn{2}{|c|}{$\begin{array}{c}\text { Línea de pobreza extrema } \\
\text { (US } \$ \text { mensuales) } 2003^{1}\end{array}$} \\
\hline & & & & or hogar & por persona \\
\hline \multirow{2}{*}{$\begin{array}{l}\text { Hogares pobres con } \\
\text { niños menores de } \\
17 \text { años (incluye } \\
\text { poblaciones } \\
\text { indígenas) }\end{array}$} & \multicolumn{3}{|c|}{$\begin{array}{l}\text { Beneficio único y simple: } 50 \text { US } \$ \text { mensuales. Fijo al hogar } \\
\text { beneficiario (frecuencia bimestral) }\end{array}$} & & \\
\hline & \multicolumn{3}{|c|}{$\begin{array}{l}\text { Transferencia en especie a hogares indígenas (Bono de } \\
\text { SENAPAN) }\end{array}$} & 312 & 45 \\
\hline
\end{tabular}

\begin{tabular}{|c|c|c|}
\hline \multicolumn{3}{|c|}{ Condicionalidad } \\
\hline Ámbito & Colectivo & Condición \\
\hline \multirow{3}{*}{ Salud } & Niños hasta 12 meses de edad & Controles de crecimiento y desarrollo \\
\hline & Mujeres embarazadas & Controles prenatales \\
\hline & $\begin{array}{c}\text { Niños entre } 0 \text { y } 4 \text { años, discapacitados y } \\
\text { adultos mayores de } 60 \text { años }\end{array}$ & Controles de salud y crecimiento \\
\hline \multirow[t]{2}{*}{ Educación } & Niños entre 4 y 17 años & $\begin{array}{c}\text { Matrícula y asistencia escolar (pre-maternal, maternal, } \\
\text { primaria y pre-media) }\end{array}$ \\
\hline & Tutores responsables del hogar & Asistencia a reuniones convocadas por el docente \\
\hline $\begin{array}{l}\text { Acompañamien } \\
\text { to familiar }\end{array}$ & Adulto miembro del hogar & Participación en talleres y actividades de capacitación \\
\hline
\end{tabular}

\begin{tabular}{|c|c|}
\hline & Sanciones \\
\hline Ámbito & \multirow{3}{*}{$\begin{array}{l}\text { El incumplimiento por parte de un miembro implica una penalización máxima de } 10 \text { dólares por bimestre. La } \\
\text { cuantía se incrementará hasta el tercer pago consecutivo. }\end{array}$} \\
\hline Salud & \\
\hline $\begin{array}{c}\text { Acompañamien } \\
\text { to familiar }\end{array}$ & \\
\hline Educación & Se permite hasta un 10 por ciento de inasistencia con respecto a los días efectivos de clases. \\
\hline
\end{tabular}
Recertificación y salida

3 años (36 meses); máximo cuatro años. Automáticamente se gradúan y salen del Programa. La recertificación puede realizarse por un período igual o menor.

${ }^{1}$ Calculada a partir de la Encuesta de Niveles de Vida de 2003. MEF

Fuente: Adaptado de Him (2017:120).

El programa Red de Oportunidades utiliza dos criterios de focalización: geográfica y por hogar. El primero permite medir la marginalidad de un corregimiento (la menor división político-administrativa de Panamá). Durante el primer año de ejecución del programa se utilizó el Índice Compuesto de Marginalidad, un índice sintético que varía entre 0 y 100 (cuanto más próximo a cero, mayor marginalidad) e incorpora dos dimensiones: (i) la pobreza extrema medida por consumo y (ii) las necesidades básicas insatisfechas de salud, vivienda, agua y saneamiento, y educación.

La focalización por hogar declara elegible a los hogares siempre que cumplan con dos criterios: (i) ser pobre extremo ${ }^{\bullet}$ de acuerdo con la definición del programa (focalización geográfica); y, (ii) cumplir determinados requerimientos de composición demográfica, distinguiendo entre áreas urbanas, rurales y comarcas indígenas, a partir del Índice Proxy Means Test (PMT), que utiliza los datos de la Encuesta de Vulnerabilidad Social (EVS) y permite captar las características socioeconómicas, demográficas, físicas y humanas de los hogares elegibles y estimar la probabilidad de que un hogar sea pobre extremo. Este procedimiento técnico busca minimizar los errores de exclusión y de inclusión al objeto de hacer llegar las transferencias a los más necesitados.

El proceso termina con la selección final de beneficiarios a partir de la probabilidad de ser pobre extremo. El punto de corte utilizado para seleccionar los hogares beneficiarios del programa Red de

\footnotetext{
- Siguiendo la metodología del Ministerio de Economía y Finanzas, pobre extremo es aquel individuo que se encuentra por debajo de la línea de pobreza extrema (indigencia). Para los efectos del Programa se estimó a partir de la Encuesta de Niveles de Vida de 2003, fijándose en 312 dólares mensuales por hogar y 45 dólares mensuales por persona (Ministerio de Desarrollo Social [MIDES], 2009).
} 
Oportunidades se ha visto modificado en tres ocasiones desde su inicio, en el año 2006, hasta la fecha, y en las tres áreas geográficas consideradas ${ }^{\bullet}$ indígena, rural y urbana (Tabla 2).

Tabla 2

Umbrales del Proxy Means Test para ser beneficiario de las transferencias del programa Red de Oportunidades

\begin{tabular}{cccc}
\hline Áreas & $\mathbf{2 0 0 6}$ & $\mathbf{2 0 0 9}$ & Frontera actual \\
\hline Indígena & Mayor o igual al 20\% & Mayor o igual al 20\% & Mayor o igual al 15\% \\
Rural & Mayor o igual al 30\% & Mayor o igual al 25\% & Mayor o igual al 25\% \\
Urbana & Mayor o igual al 40\% & Mayor o igual al 35\% & Mayor o igual al 35\% \\
\hline
\end{tabular}

Fuente: Adaptado de Him (2017:121).

El segundo parámetro lo constituye la estructura de beneficios. El programa Red de Oportunidades contempla únicamente una transferencia monetaria (homogénea y fija por hogar) en efectivo, sin que ello suponga que los beneficiarios tengan que renunciar a otras transferencias en especie ${ }^{\bullet}$. La cuantía inicialmente fijada fue de 35 dólares mensuales (pagos bimestrales), sin embargo, desde el año 2008, esta cantidad se incrementa hasta los 50 dólares mensuales con la intención de contrarrestar el impacto de la crisis mundial. Como excepción, las comarcas indígenas reciben el pago por familia y no por hogar. Al igual que otros programas de América Latina y el Caribe, la persona responsable de recibir la transferencia es la madre o, en su defecto (no existe, está imposibilitada o carece de cédula de identidad), la persona responsable del hogar.

Las condicionalidades o corresponsabilidades son uno de los parámetros más complejos de instrumentar, dado que requiere de una estructura administrativo-institucional sólida que vigile su cumplimiento. El programa Red de Oportunidades contempla tres ámbitos en este aspecto: salud, educación y acompañamiento familiar.

La condicionalidad de salud se aplica a todos los hogares beneficiarios que tengan niños de 0 a 4 años, mujeres embarazadas, adultos mayores de 60 años y/o personas con discapacidad. Deben cumplir con controles periódicos prenatales, postnatales, de crecimiento, inmunizaciones y desarrollo, en función del grupo al que pertenezcan. Su incumplimiento por parte de uno de los miembros del hogar beneficiario implica una penalización máxima de 10 dólares por bimestre, incrementándose esta cuantía hasta el tercer pago consecutivo.

La condicionalidad de educación se aplica a los hogares beneficiarios con niños de entre 4 y 17 años de edad, que deben matricularse en el sistema educativo formal o no formal aprobado por el Ministerio de Educación (MEDUCA) y asistir regularmente a clase. El programa fija una serie de excepciones a las corresponsabilidades en educación para aquellos niños que en el momento de la inscripción demuestren: padecer alguna discapacidad o enfermedad crónica; vivir alejados del centro educativo disponible (más de dos horas de viaje o un coste adicional de 10 dólares al mes); problemas de seguridad; que el centro educativo disponible no tenga capacidad adicional para aceptar niños beneficiarios o no exista oferta educativa disponible en el área de influencia. Se permite hasta un 10 por ciento de inasistencia con respecto a los días efectivos de clase (MIDES, 2009).

El programa Red de Oportunidades también contempla una estrategia de acompañamiento familiar, con el fin de apoyar a las familias beneficiarias, que se materializa en la asistencia a talleres de formación organizados por equipos técnicos para dar a conocer sus derechos, fortalecer el capital humano y social de las comunidades y lograr un desarrollo óptimo de sus potencialidades. El sistema de sanciones a su incumplimiento es similar al que se establece para la condicionalidad en salud. Esta estrategia ha tenido un desarrollo escaso y desigual en las diferentes provincias y comarcas indígenas del país (Gómez Hermosillo, 2009).

\footnotetext{
- A los efectos de esta investigación, los cálculos se han realizado con los puntos de corte actualmente vigentes, con una probabilidad mayor o igual del 35 por ciento para las áreas urbanas; mayor o igual del 25 por ciento para las rurales; y, mayor o igual del 15 por ciento para las comarcas indígenas.

- Como es el caso de los beneficiarios del programa Red de Oportunidades de las comarcas indígenas que reciben un bono para la compra de alimentos de la Secretaría Nacional para el Plan Alimentario (SENAPAN).
} 
Finalmente, el último de los parámetros se refiere a los criterios de recertificación y salida de los beneficiarios. El programa fija un período de permanencia de tres años (36 meses) pudiendo extenderse hasta un máximo de cuatro. Culminado este período, los beneficiarios se gradúan y salen definitivamente de él. Sin embargo, podrían someterse a un proceso de recertificación por un periodo igual o menor si lo considera oportuno el gestor del programa. La definición de este parámetro presenta ambigüedades que podrían ser objeto de discrecionalidades administrativas. No está claro si la salida de un beneficiario obedece a la superación de su condición de pobreza, a ajustes presupuestarios o a razones de clientelismo político.

\subsection{Articulación institucional del programa}

La responsabilidad última del programa Red de Oportunidades descansa en el Gabinete Social, que toma las decisiones político-estratégicas y está integrado por cuatro ministerios (Figura 1): el Ministerio de Desarrollo Social (MIDES) que lo preside; el Ministerio de Economía y Finanzas (MEF); el Ministerio de Educación (MEDUCA); y el Ministerio de Salud (MINSA). La Contraloría General de la República participa a nivel superior como organismo fiscalizador del Fondo Especial para la Pobreza Extrema responsable último de hacer llegar a los beneficiarios las transferencias monetarias condicionadas.

\section{Figura 1}

Articulación institucional del programa Red de Oportunidades

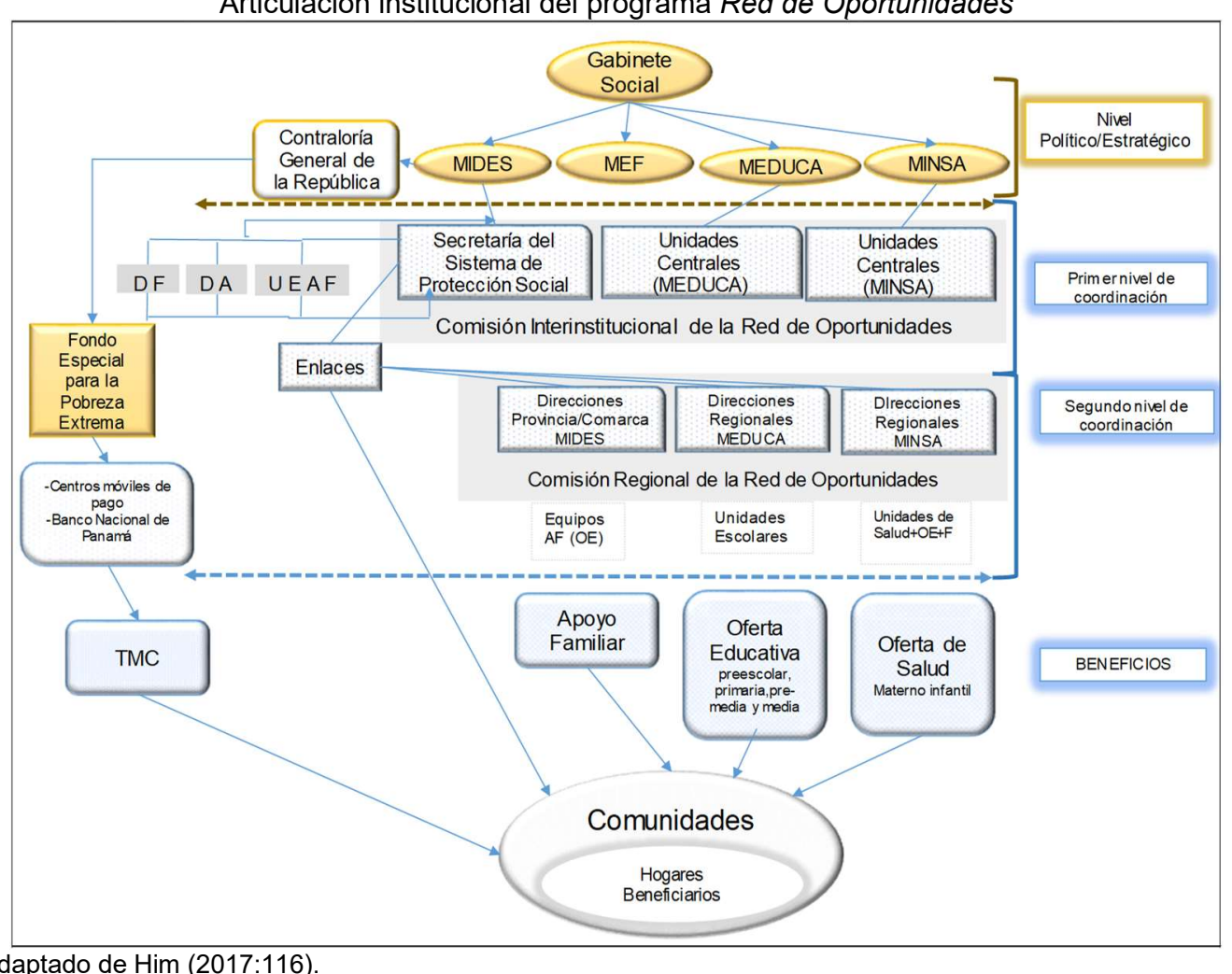

Fuente: Adaptado de Him (2017:116).

La estructura contempla dos niveles de coordinación. En el primero se encuentra la Comisión Interinstitucional de la Red de Oportunidades integrada por la Secretaría del Sistema de Protección Social (SSPS) y dos unidades centrales de los Ministerios que intervienen directamente en el cumplimiento de las corresponsabilidades (MEDUCA y MINSA). A su vez, esta Comisión se apoya en tres Direcciones del MIDES: la Dirección de Finanzas (DF); la Dirección Administrativa (DA); y la Unidad de Enlace Administrativo-Financiero (UEAF). En el segundo nivel intervienen los enlaces de las Direcciones Provinciales/Regionales de los tres Ministerios involucrados en la intervención (Comisión Regional de la Red de Oportunidades, CPRO) (Him, Ogando y Miranda, 2016). 
Los beneficios del programa se encuentran en el último nivel y deben llegar a todas las comunidades $\mathrm{y}$ hogares beneficiarios. Incluyen cuatro aspectos: el pago de las transferencias monetarias condicionadas; el apoyo familiar; la oferta educativa; y la oferta de salud.

El Manual Operativo del programa (MIDES, 2009) establece los mecanismos para la verificación y cumplimiento de las corresponsabilidades de las transferencias monetarias condicionadas, otorgando un papel prioritario en esta tarea a las Direcciones Regionales del MEDUCA y del MINSA. En el primero de los casos, estas direcciones tienen como cometido ejecutar el programa a nivel regional, actuando como enlace entre la Comisión Institucional del Ministerio de Educación, los centros educativos y la población beneficiaria. Son precisamente los centros educativos los que tienen que recabar la información requerida, así como rellenar los formularios indicando si los niños beneficiarios fueron admitidos en la unidad escolar y si están asistiendo regularmente a clase. En el segundo, las Direcciones Regionales del MINSA constituyen el vínculo entre la Comisión Institucional del Ministerio de Salud, las unidades básicas de atención en salud y la población beneficiaria. Estas unidades informan a las respectivas Direcciones Provinciales/Comarcales del MIDES del cumplimiento de la corresponsabilidad en salud.

Se trata de un esquema de intervención complejo que compromete una multiplicidad de instituciones y funcionarios con una excesiva burocracia y sin una delimitación clara de tareas y competencias. Dado que algunas de las evaluaciones realizadas (Waters, 2009a, 2009b; Gómez Hermosillo, 2009) alertan sobre las deficiencias en la articulación institucional del programa, particularmente en el cumplimiento de las corresponsabilidades en salud y educación, así como en las limitaciones de la oferta y la calidad de estos servicios, una cuestión a investigar es si existen mecanismos adecuados de coordinación y si se ha realizado un diagnóstico real de los servicios educativos y sanitarios que permita la eficaz ejecución de una política de estas características.

\section{METODOLOGÍA Y DATOS}

\subsection{Metodología}

No existe un método único que pueda proporcionar por sí solo la evidencia que se busca, pero la elección adecuada de una combinación de técnicas de evaluación (basadas en teorías, cualitativas, cuantitativas o mixtas) y métodos (experimentales, cuasi-experimentales o no experimentales) pueden ayudar a la hora de emitir juicios sólidos sobre un programa (European Commission, 2014). Unas técnicas pueden complementarse con otras obteniendo así mejores y más útiles evaluaciones, siendo recomendable un mix en el uso de métodos y técnicas de evaluación para obtener múltiples perspectivas de un mismo programa.

Las técnicas cuantitativas tienen como objetivo mejorar la medición de los impactos de un programa, responden a la pregunta "cuánto" y su resultado es un número. Las cualitativas intentan averiguar "cómo" y "por qué" unas intervenciones funcionan y otras no, siendo su resultado un relato. A pesar de los inconvenientes que plantea el uso de estas últimas (Weiss, 1987), posibilitan la participación de muchos stakeholders con experiencias distintas sobre la intervención, ofreciendo puntos de vista diferentes que permiten obtener una visión multisectorial de los factores de éxito y fracaso del programa (Miranda, Rojo, Gómez y Cavero, 2015). Requieren la recopilación de evidencias mediante encuestas, entrevistas estructuradas, focus groups, estudios de caso, o revisando y analizando información administrativa y documental. Todas ellas han sido utilizadas en esta investigación ${ }^{\bullet}$. Una vez que se dispone de esta evidencia empírica, se pueden formular conclusiones robustas sobre las relaciones causales que pueden explicar cómo las actividades de la intervención generan una serie de resultados que contribuyen a lograr los impactos finales previstos, lo que se conoce como teoría del cambio (Rogers, 2014).

\footnotetext{
- Un análisis detallado de las características, las ventajas y los inconvenientes de la aplicación de este tipo de técnicas para evaluar la gobernanza de un programa público puede consultarse en Him (2017).
} 


\subsection{Datos}

Los datos utilizados en esta investigación proceden de diversas fuentes. En primer lugar, los obtenidos del Sistema de Información Gerencial $(S I G)$ a partir de los registros de los beneficiarios del programa (424.716 individuos y 73.139 hogares en enero de 2015) que han sido proporcionados por el Ministerio de Desarrollo Social de Panamá. Esta información ha sido complementada con otra, de carácter administrativo y documental, que también ha sido facilitada por el órgano gestor de la Red de Oportunidades. Además, durante los meses de agosto y septiembre de 2015, se llevó a cabo un trabajo de campo que ha consistido en la realización de una serie de entrevistas, mediante cuestionarios semiestructurados, a diferentes actores del programa.

Se efectuaron 9 entrevistas personales "in situ" a determinadas autoridades del Ministerio de Desarrollo Social y a la Secretaría del Sistema de Protección Social (SSPS), unidad encargada de la gestión del programa ${ }^{\bullet}$. El objetivo era identificar a los actores que intervienen durante el proceso para recabar información y documentación de las personas beneficiarias o de sus representantes.

Se realizó un muestreo intencional y se lanzaron 96 cuestionarios de tres tipos diferentes dirigidos a técnicos responsables de la gestión del programa y a personas indirectamente vinculadas a él, como los Directores de Centros Escolares, con la distribución que se presenta en la (Tabla 3). Dado que el programa cubre tres tipos de áreas geográficas (indígena, rural y urbana), con cerca de medio millón de beneficiarios a escala nacional, fue necesario acotar el ámbito de estudio al medio rural de las Provincias Centrales porque presentan unas características socioeconómicas más homogéneas. La aplicación de estos cuestionarios se realizó, bien a través de entrevistas personales, bien mediante dinámicas grupales $\mathrm{y}$, en menor medida, por correo electrónico.

Tabla 3

Cuestionarios distribuidos y grado de respuesta

\begin{tabular}{lccc}
\hline \multicolumn{1}{c}{ Cuestionarios } & Total & Respondidos & Porcentaje \\
\hline Cuestionario 1: Nivel Político-Estratégico & 12 & 2 & 16,7 \\
Cuestionario 2: Nivel Técnico-Operativo & 44 & 30 & 68,2 \\
Cuestionario 3: Directores de Centros Escolares & 40 & 21 & 52,5 \\
$\quad$ Totales & 96 & 53 & 55,2 \\
\hline
\end{tabular}

Fuente: Adaptado de Him (2017:104).

Se obtuvo un nivel de respuesta del 55,2\%. El grupo que menos contestó fue el correspondiente al nivel político-estratégico y, el que lo hizo en mayor medida, el de los gestores técnicos operativos. Asimismo, el 68,8\% de los Directores de Centros Escolares de la Provincia de Veraguas contestaron, obteniéndose el menor nivel de respuesta en los de la provincia de Coclé $(27,3 \%)$ (Tabla 4).

Tabla 4

Cuestionarios distribuidos en Centros Escolares de las Provincias Centrales

\begin{tabular}{cccc}
\hline Provincias Centrales & Total & Respuestas & Porcentaje \\
\hline Coclé & 11 & 3 & 27,3 \\
Herrera & 6 & 3 & 50,0 \\
Los Santos & 7 & 4 & 57,1 \\
Veraguas & 16 & 11 & 68,8 \\
Total & 40 & 21 & 52,5 \\
\hline
\end{tabular}

Fuente: Adaptado de Him (2017:104).

No se puede perder de vista el carácter subjetivo de las valoraciones y apreciaciones de cada uno de los gestores y Directores de Centros Escolares encuestados. Por este motivo, para complementar el análisis cualitativo y la interpretación de los resultados, se han utilizado datos administrativos procedentes del Sistema de Información Gerencial del programa. A esta información se le ha añadido

\footnotetext{
- Se mantuvo una entrevista con la Secretaría del Sistema de Protección Social (SSPS) y técnicos de diferentes Departamentos tales como: Unidad de Soporte de Tecnología e Informática; Monitoreo y Evaluación; Unidad de Corresponsabilidades (educación y salud); Unidad Administrativa y Asesoría Legal; Unidad de Enlace Administrativo-Financiero (UEAF); Directores de Centros Escolares que acogen niños y niñas beneficiarios de la Red de Oportunidades; Directores Regionales del MIDES; y, Coordinadores del programa Red de Oportunidades en las Provincias Centrales (Coclé, Herrera, Los Santos y Veraguas).
} 
otra de carácter administrativo, documental y financiera, también facilitada por el órgano gestor de la intervención.

Asimismo, se ha utilizado la Encuesta Continua a Hogares (ECH) del Instituto de Estadística y Censo de Panamá (años 2010 y 2013), que se elabora de forma ininterrumpida desde el año 1963. La muestra que utiliza esta encuesta se extrae del Censo de Población y Vivienda del año 2000 y el universo está constituido por la población de 15 y más años de edad que reside en viviendas particulares. La información suministrada es representativa a nivel de provincias y comarcas indígenas, así como de áreas urbanas y rurales. En el año 2010, la muestra incluye un total de 48.881 personas que pertenecen a 13.391 hogares; en 2013 estos valores fueron 44.237 y 11.853 , respectivamente.

\section{RESULTADOS}

\subsection{Participación de los actores implicados en el programa y mecanismos de coordinación}

El éxito de un programa público depende del acierto en su formulación, de la voluntad política, de la capacidad para alcanzar acuerdos, para formar coaliciones con grupos sociales y para propiciar cambios institucionales, políticos y organizacionales. Desde la perspectiva del desarrollo humano, la participación de los actores también resulta relevante porque lograr mejores condiciones de vida y la participación en la sociedad de la que se forma parte no son prácticas exclusivamente personales; los actores sociales pueden contribuir a la colectivización de las aspiraciones y experiencias individuales (Programa de Naciones Unidas para el Desarrollo [PNUD], 2015).

En el trabajo de campo realizado en la investigación se intentó determinar en qué grado habían participado los agentes socioeconómicos en el diseño del programa. Las respuestas obtenidas identifican tres actores que lo hicieron en mayor medida: el Gabinete Social, expertos internacionales y los gobiernos locales (representantes de los corregimientos, la unidad político-administrativa más pequeña del país). El resto tuvo un papel muy limitado (Figura 2).

Además, los gestores señalaron que el diseño de la estrategia siguió un enfoque de "arriba a abajo" (top-down). Aunque consideraban que no es el método más adecuado, hubo que implementarlo debido a la necesidad de cumplir con los términos y condiciones exigidas por las instituciones financieras internacionales (BID y BM), motivo por el cual se omitieron algunos procesos, en especial la consulta a otros grupos, beneficiarios y sociedad civil en general, que podrían haber aportado información relevante para la intervención. De hecho, la literatura (Barrientos, Gideon y Molyneux, 2008) reconoce que este tipo de políticas está fuertemente influenciado por estas dinámicas: la intervención se gestiona desde las élites políticas hacia las unidades administrativas más bajas.

\section{Figura 2}

Participación de los agentes socioeconómicos en el diseño del programa Red de Oportunidades (Porcentaje de respuestas)



Fuente: Adaptado de Him (2017:115). 
Por otro lado, la gran mayoría de los equipos gestores del programa consultados $(91,3 \%)$ manifestaron que existe una clara delimitación de funciones entre los distintos departamentos de la Secretaría del Sistema de Protección Social (SSPS), con una estructura administrativo-operativa que facilita la implementación de los procedimientos a seguir, no produciéndose conflictos de competencias (Tabla 5).

Tabla 5

Valoración de los gestores sobre la división de tareas y competencias entre los actores institucionales del programa

\begin{tabular}{lcc}
\hline \multicolumn{1}{c}{ Funciones y competencias } & \multicolumn{2}{c}{ Respuestas (\%) } \\
\cline { 2 - 3 } & \multicolumn{1}{c}{ Sí } & No \\
\hline 1. Existencia de funciones claramente delimitadas & 91,3 & 8,7 \\
2. Correcto funcionamiento de los mecanismos de coordinación entre: & 56,9 & 43,1 \\
2.1. Los departamentos del SSPS & 58,7 & 41,3 \\
2.2. Distintas Comisiones (CIRO y CPRO) & 65,2 & 34,8 \\
2.3. La Comisión Regional de la Red de MEDUCA y MINSA & 65,2 & 34,8 \\
2.4. Las direcciones provinciales y la Comisión Interinstitucional (CIRO) & 55,6 & 44,4 \\
2.5. Los promotores sociales y los beneficiarios & 54,3 & 45,7 \\
2.6. Los promotores sociales y los centros escolares & 42,5 & 57,5 \\
3. Suficientes recursos humanos y materiales para alcanzar los objetivos & 25,5 & 74,5 \\
\hline
\end{tabular}
Fuente: Adaptado de Him (2017:115).

Sin embargo, en cuanto a los mecanismos de coordinación, las respuestas obtenidas no fueron tan contundentes como en el caso anterior. Los gestores admitieron que existen mecanismos de carácter formal entre los distintos agentes implicados en la gestión y ejecución del programa (entre departamentos del SSPS, entre las distintas comisiones interinstitucionales, entre los diferentes ministerios involucrados a nivel regional y entre los promotores sociales y los beneficiarios), siendo satisfactoria la valoración que efectuaron sobre su funcionamiento para el 56,9\% de los encuestados. Además, reconocieron que se establecen colaboraciones con otros ministerios e instituciones (por ejemplo, el Instituto Nacional para la Formación y Capacitación para el Desarrollo-INADEH y la Autoridad de la Micro, Pequeña y Mediana Empresa-AMPYME), que organizan cursos de formación y talleres dirigidos a los beneficiarios del programa para promover el espíritu empresarial.

Ahora bien, se detectaron problemas de coordinación entre los promotores sociales y los centros escolares, por un lado (así lo señala el 57,5\% de los consultados), y entre aquéllos y los beneficiarios directos, por otro (el 45,7\% de los encuestados). Estos resultados confirman que una de las debilidades de la coordinación institucional se sitúa en el último eslabón de la cadena, entre los promotores sociales con los beneficiarios y con las instituciones colaboradoras de la intervención (los centros escolares).

No obstante, si atendemos a las respuestas ofrecidas por tipo de encuestado (Técnicos-operativos, por un lado, y Directores de Centros Escolares, por otro), se observan diferencias significativas en las valoraciones (Figura 3).

Los técnicos del MIDES situaron por encima de la media el funcionamiento de todas las modalidades de coordinación (entre departamentos, comisiones y ministerios involucrados), de cara al cumplimiento de la corresponsabilidad en educación. En cambio, los Directores de Centros Escolares proporcionaron valoraciones muy por debajo de la media, excepto para la coordinación que se realiza a nivel provincial con MEDUCA y la Comisión Regional del Programa (CPRO). 
Figura 3

Valoración media de las diferentes modalidades de coordinación: Técnicos-operativos versus Directores de Centros Escolares

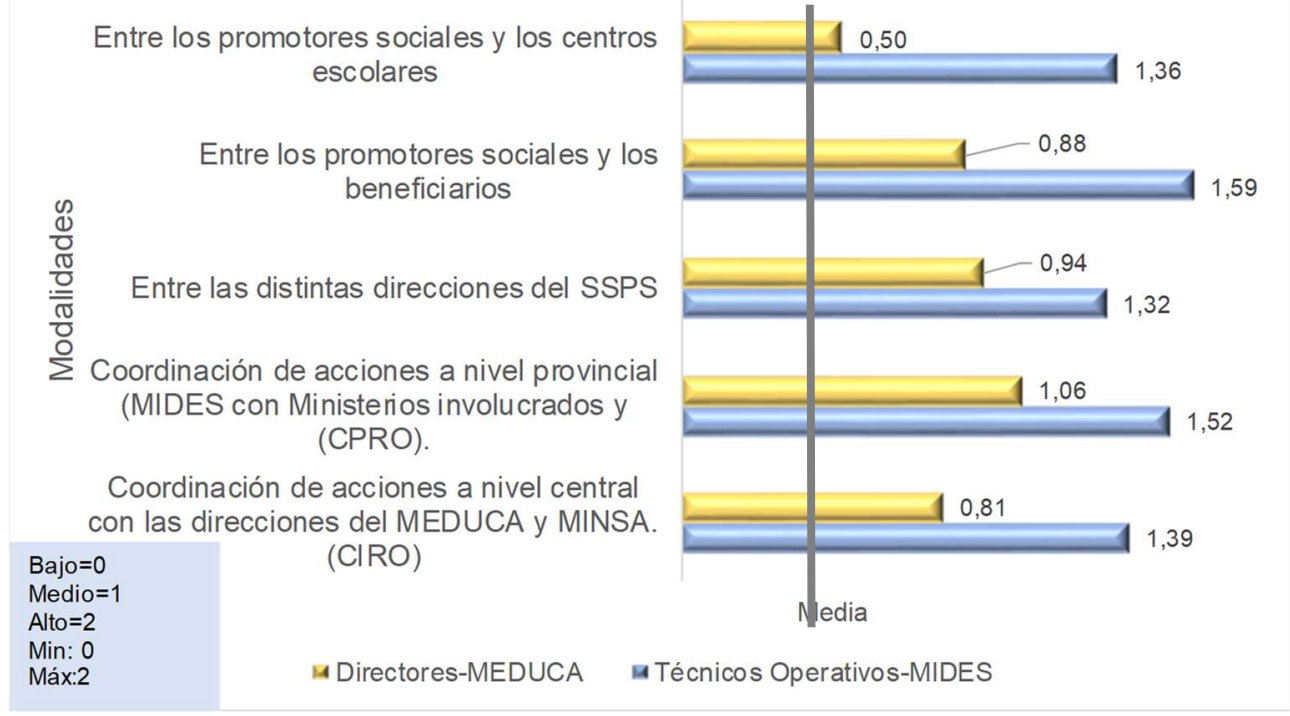

Fuente: Adaptado de Him (2017:119).

\subsection{Aplicación de los principales parámetros del programa: criterios de selección de los beneficiarios y control de las corresponsabilidades}

Durante la realización del trabajo de campo se quiso pulsar la opinión de los gestores sobre la aplicación de los principales parámetros del programa. Valoraron satisfactoriamente el acompañamiento familiar $(66,7 \%)$ y el cumplimiento de la corresponsabilidad en salud $(48,2 \%)$, mientras que la de educación solo tuvo esta consideración para el $37 \%$ de los encuestados. Las peores valoraciones se obtuvieron para los mecanismos de salida y recertificación de beneficiarios $(81,5 \%)$, la gestión de las reclamaciones $(80,8 \%) \mathrm{y}$, en menor medida, la selección de beneficiarios $(59,3 \%)$ (Tabla $6)$.

Tabla 6

Valoración de los gestores sobre la aplicación de los parámetros del programa

\begin{tabular}{|c|c|c|c|c|c|}
\hline \multirow{2}{*}{ Parámetros } & \multicolumn{5}{|c|}{ Valoración (\%) } \\
\hline & Muy mala & Mala & Regular & Buena & Muy Buena \\
\hline Selección de beneficiarios & 22,22 & 7,41 & 29,63 & 22,22 & 18,52 \\
\hline Salida y recertificación de beneficiarios & 25,93 & 14,81 & 40,74 & 11,11 & 7,41 \\
\hline Gestión de reclamaciones & 19,23 & 15,38 & 46,15 & 19,23 & 0 \\
\hline \multicolumn{6}{|l|}{ Cumplimiento de corresponsabilidades } \\
\hline Educación & 22,22 & 3,70 & 37,04 & 25,93 & 11,11 \\
\hline Salud & 22,22 & 14,81 & 14,81 & 37,04 & 11,11 \\
\hline Acompañamiento familiar & 22,22 & 3,70 & 7,41 & 44,44 & 22,22 \\
\hline
\end{tabular}

Fuente: Elaboración propia a partir de los resultados de las entrevistas.

Las respuestas de los gestores en este último aspecto se corroboran con el análisis efectuado a partir de la información suministrada por el Sistema de Información Gerencial del programa, en relación con los criterios de selección de los beneficiarios.

La Figura 4 muestra la cobertura y distribución geográfica de los 424.716 beneficiarios que había registrados a 31 de diciembre de 2014, con presencia en 619 corregimientos de los 631 en los que se divide el país, si bien con una mayor concentración en las áreas rurales (color azul) y sobre todo en las Provincias Centrales. 


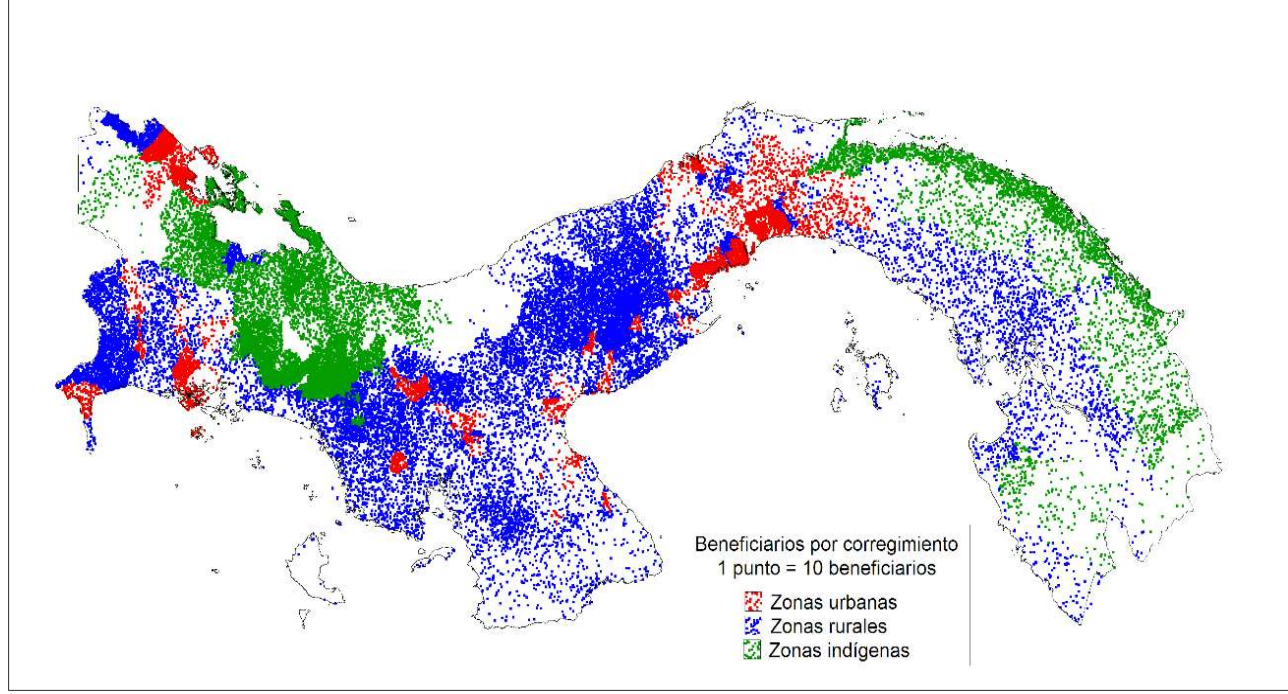

Fuente: Adaptado de Him (2017:122).

Se ha detectado una filtración del 13,68\% de beneficiarios incluidos en las tres áreas geográficas (un total de 58.082) que no deberían haber sido asistidos (errores de inclusión), mientras que el 86,32\% calificaron en las distintas probabilidades de "ser pobre extremo". Al considerar el conjunto de no beneficiarios (452.062 personas que solicitaron la transferencia y que no fueron elegidas), los resultados revelaron que existe una filtración del 9,61\% de solicitantes excluidos, en las tres áreas geográficas consideradas, con una probabilidad mayor o igual al 35\% de "ser pobre extremo", lo que significa que 43.461 personas que deberían haber sido asistidas por el programa quedaron fuera de él (errores de exclusión) (Tabla 7).

Tabla 7

Beneficiarios y no beneficiarios del programa a partir del proxy means test a 31 de diciembre de 2014

\begin{tabular}{|c|c|c|c|c|c|c|c|}
\hline & $\begin{array}{l}\text { Probabil } \\
\text { idad }\end{array}$ & $\begin{array}{l}\text { Indíg } \\
\text { ena }\end{array}$ & $\underset{I}{\text { Rura }}$ & $\begin{array}{c}\text { Urba } \\
\text { no }\end{array}$ & Total & $\begin{array}{c}\% \text { de incluidos que satisfacen } \\
\text { el criterio }\end{array}$ & $\begin{array}{c}\% \text { de incluidos que no satisfacen el } \\
\text { criterio y participan }\end{array}$ \\
\hline \multirow{7}{*}{ 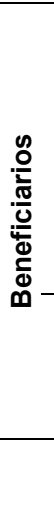 } & $<0,15$ & 692 & $\begin{array}{c}14.5 \\
82\end{array}$ & $\begin{array}{c}6.29 \\
3\end{array}$ & $\begin{array}{c}21.5 \\
67\end{array}$ & & 5,08 \\
\hline & $\begin{array}{c}0,15- \\
0,25\end{array}$ & 2.014 & $\begin{array}{c}26.6 \\
40\end{array}$ & $\begin{array}{c}3.06 \\
9\end{array}$ & $\begin{array}{l}31.7 \\
23\end{array}$ & 0,47 & 7,00 \\
\hline & $\begin{array}{c}0,25- \\
0,35\end{array}$ & 3.068 & $\begin{array}{c}43.7 \\
30\end{array}$ & $\begin{array}{c}6.80 \\
6\end{array}$ & $\begin{array}{c}53.6 \\
04\end{array}$ & 11,02 & 1,60 \\
\hline & $>0,35$ & $\begin{array}{c}147.1 \\
76\end{array}$ & $\begin{array}{l}119 . \\
454\end{array}$ & $\begin{array}{c}51.1 \\
92\end{array}$ & $\begin{array}{l}317 . \\
822\end{array}$ & 74,83 & \\
\hline & Total & $\begin{array}{c}152.9 \\
50\end{array}$ & $\begin{array}{l}204 . \\
406\end{array}$ & $\begin{array}{c}67.3 \\
60\end{array}$ & $\begin{array}{l}424 . \\
716\end{array}$ & & \\
\hline & (\%) & 36,01 & $\begin{array}{c}48,1 \\
3\end{array}$ & $\begin{array}{c}15,8 \\
6\end{array}$ & $\begin{array}{c}100 \\
00\end{array}$ & 86,32 & 13,68 \\
\hline & $\begin{array}{l}\text { Probabil } \\
\text { idad }\end{array}$ & $\begin{array}{l}\text { Indíg } \\
\text { ena }\end{array}$ & $\underset{I}{\text { Rura }}$ & $\begin{array}{c}\text { Urba } \\
\text { no }\end{array}$ & Total & $\begin{array}{l}\% \text { de excluidos que no } \\
\text { satisfacen el criterio }\end{array}$ & $\begin{array}{l}\% \text { de excluidos que satisfacen el criterio } \\
\text { pero no participan }\end{array}$ \\
\hline \multirow{6}{*}{ 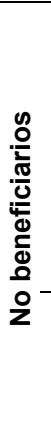 } & $<0,15$ & 885 & $\begin{array}{l}233 . \\
879\end{array}$ & $\begin{array}{l}130 . \\
631\end{array}$ & $\begin{array}{l}365 . \\
395\end{array}$ & 80,83 & \\
\hline & $\begin{array}{c}0,15- \\
0,25\end{array}$ & 563 & $\begin{array}{c}29.5 \\
39\end{array}$ & $\begin{array}{c}9.84 \\
4\end{array}$ & $\begin{array}{l}39.9 \\
46\end{array}$ & 8,71 & 0,12 \\
\hline & $0,25-$ & 738 & $\begin{array}{c}7.17 \\
9\end{array}$ & $\begin{array}{c}3.82 \\
3\end{array}$ & $\begin{array}{l}11.7 \\
40\end{array}$ & 0,85 & 1,75 \\
\hline & $>0,35$ & $\begin{array}{c}18.62 \\
3\end{array}$ & $\begin{array}{c}13.5 \\
32\end{array}$ & $\begin{array}{c}2.82 \\
6\end{array}$ & $\begin{array}{c}34.9 \\
81\end{array}$ & & 7,74 \\
\hline & Total & $\begin{array}{c}20.80 \\
9\end{array}$ & $\begin{array}{l}284 . \\
129\end{array}$ & $\begin{array}{l}147 . \\
124\end{array}$ & $\begin{array}{l}452 . \\
062\end{array}$ & & \\
\hline & (\%) & 4,60 & $\begin{array}{c}62,8 \\
5\end{array}$ & $\begin{array}{c}32,5 \\
5\end{array}$ & $\begin{array}{c}100 \\
00\end{array}$ & 90,39 & 9,61 \\
\hline
\end{tabular}


Para contrastar la robustez de los resultados anteriores, se ha comparado la distribución del ingreso de los hogares antes y después de recibir las transferencias a partir de los datos de la Encuesta Continua a Hogares (ECH) del Instituto de Estadística y Censo de Panamá (años 2010 y 2013) (Tabla 8). En el año 2010, la transferencia supuso un incremento del 14,45\% para los hogares que se encuentran en el primer quintil de ingresos. En otros términos, la razón ingreso con transferencias mejoró 2,5 puntos, con respecto a la razón de ingreso sin transferencias en ese año (Q5/Q1). En 2013, las transferencias incrementaron los ingresos del $20 \%$ más pobre en 35,30 dólares en términos absolutos, situación que mejora el ingreso medio de este grupo en un 19,43\%, aumentando la razón ingreso 2,99 puntos en ese mismo año. Los resultados ponen de manifiesto que las transferencias consiguen cierto avance en la reducción de la desigualdad, aunque apenas perceptible.

Tabla 8

Distribución del ingreso medio de los hogares según quintiles antes y después de recibir la transferencia monetaria condicionada (TMC)

\begin{tabular}{|c|c|c|c|c|c|c|c|c|c|}
\hline \multicolumn{5}{|c|}{2010} & \multicolumn{5}{|c|}{2013} \\
\hline Quintil & $\begin{array}{l}\text { con } \\
\text { TMC }\end{array}$ & $\begin{array}{l}\sin \\
\text { TMC }\end{array}$ & Dif. & $\begin{array}{c}\text { Incremento } \\
(\%)\end{array}$ & Quintil & $\begin{array}{l}\text { con } \\
\text { TMC }\end{array}$ & $\begin{array}{l}\sin \\
T M C\end{array}$ & Dif. & $\begin{array}{c}\text { Incremento } \\
(\%)\end{array}$ \\
\hline Q1 & 150,70 & 131,67 & 19,03 & 14,45 & Q1 & 217,02 & 181,72 & 35,30 & 19,43 \\
\hline Q2 & 364,33 & 356,67 & 7,66 & 2,15 & Q2 & 520,59 & 496,62 & 23,97 & 4,83 \\
\hline Q3 & 612,37 & 608,98 & 3,39 & 0,56 & Q3 & 853,59 & 833,84 & 19,75 & 2,37 \\
\hline Q4 & $1.006,26$ & $1.004,52$ & 1,74 & 0,17 & Q4 & $1.357,56$ & $1.336,94$ & 20,62 & 1,54 \\
\hline Q5 & $2.583,79$ & $2.586,47$ & $-2,68$ & $-0,10$ & Q5 & $3.445,49$ & $3.427,70$ & 17,79 & 0,52 \\
\hline Total & 943,31 & 937,14 & 6,17 & 0,66 & Total & $1.278,31$ & $1.254,49$ & 23,82 & 1,90 \\
\hline $\begin{array}{l}\text { Razón } \\
\text { Q5/Q1 }\end{array}$ & 17,14 & 19,64 & & & $\begin{array}{l}\text { Razón } \\
\text { Q5/Q1 }\end{array}$ & 15,88 & 18,86 & & \\
\hline
\end{tabular}

Fuente: Adaptado de Him (2017:189).

La Figura 5 ilustra la distribución del ingreso de los hogares que participan y no participan en la Red de Oportunidades en los años 2010 y 2013. El eje de abscisas mide el ingreso de los hogares hasta el quintil 4 y el de ordenadas la función de densidad (número de personas para cada nivel de ingresos). En el año 2010, se observa la concentración de un grupo de individuos cuyos ingresos se encuentran por debajo de los 642 dólares (Q1 y Q2) y que no participan del programa (error de exclusión), así como individuos en los quintiles de ingreso más altos que reciben la ayuda (error de inclusión). La distribución de ingresos para el año 2013 no presenta cambios significativos a pesar de que la cola superior de la distribución indica un incremento en la cantidad de participantes cuyos ingresos son superiores a los umbrales de pobreza, produciéndose así una filtración del programa hacia hogares que no deberían beneficiarse de él.

\section{Figura 5}

Distribución del ingreso medio de los hogares según su participación en el programa Red de Oportunidades
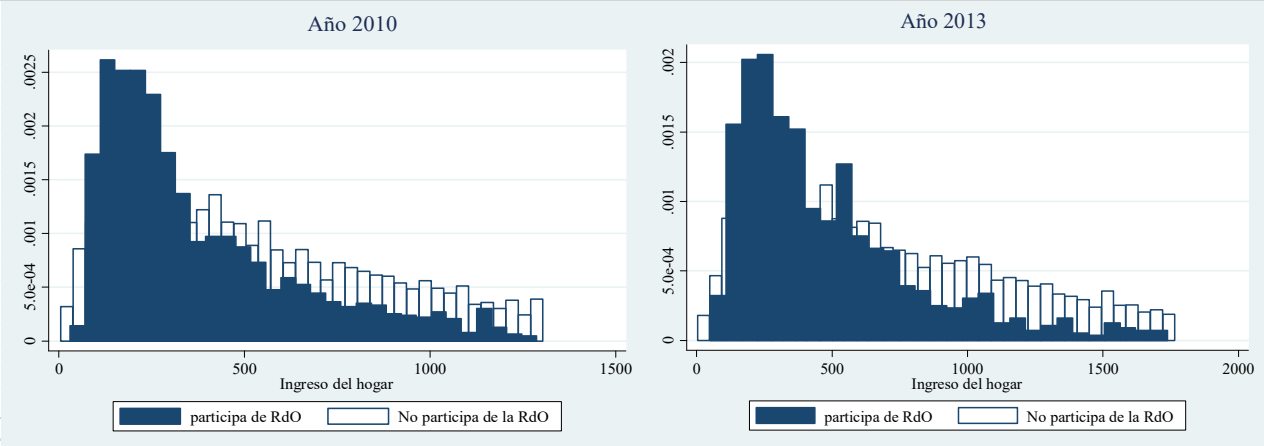

Fuente: Adaptado de Him (2017:189).

Los resultados obtenidos están de acuerdo con otras investigaciones (Muller y Bibi, 2010) que señalan que esta metodología sólo es apropiada para focalizar a los pobres crónicos y no a quienes se encuentran muy cerca de los umbrales de pobreza. Por su parte, Kidd y Wylde (2011) también destacan 
la existencia de errores de inclusión y exclusión de beneficiarios en el diseño e implementación del proxy means test, errores que, por otra parte, se ven a menudo afectados por el ciclo político, el clientelismo y la complicidad de los administradores de los gobiernos de turno (Barrientos y Dejong, 2006; De Brauw y Hoddinott, 2011).

Además, esta metodología está muy influenciada por la elección de las escalas de equivalencia, así como por el criterio utilizado para determinar qué variables se incluyen en los análisis de regresión. Pequeñas diferencias, en estos ámbitos, pueden conducir a diferencias significativas entre los hogares que son identificados para formar parte del programa. Barrett y Kidd (2015) señalan que los errores tienden a ser elevados por lo difícil que resulta obtener información precisa y fiable de los hogares, siendo habitual que más de la mitad de los aspirantes queden excluidos de la asistencia.

Por otra parte, las condicionalidades o corresponsabilidades son uno de los parámetros del programa más complejos de instrumentar, dado que resulta imprescindible disponer de una estructura administrativo-institucional sólida que vigile su cumplimiento. La condicionalidad en educación exige que los niños en edad escolar de los hogares beneficiarios se matriculen en el sistema educativo formal o no formal aprobado por el Ministerio de Educación y asistir regularmente a clase. La provisión del servicio se realiza a través de los centros escolares que se registran en un listado del Ministerio con capacidad para aceptar más niños. Por su parte, los padres o tutores deben asistir a reuniones bimestrales convocadas por los docentes responsables (MIDES, 2009). El seguimiento es responsabilidad de los promotores sociales, quienes están en contacto con los beneficiarios, a partir de la información de matrícula y asistencia que suministran cada dos meses los centros escolares.

Estos dos indicadores (matrícula y asistencia escolar) son necesarios, pero no suficientes para garantizar la superación de la condición de pobreza. Los resultados de las evaluaciones realizadas muestran que la transferencia mejora el acceso a la escuela, la matrícula y la asistencia escolar, pero que los resultados de aprendizaje dependen de la cantidad y calidad de la oferta de servicios públicos (Cecchini, 2014). En este sentido, los Directores de Centros Escolares entrevistados, que manifestaron tener entre un $3 \%$ y un $55 \%$ de sus estudiantes beneficiarios del programa, consideraron que la matrícula y la asistencia escolar se habían comportado muy bien (4,10 y 3,68 de media, respectivamente, en una escala de 0 a 5) siendo las valoraciones para el resto de aspectos consultados (resultado de calificaciones y cambio de conducta) también positivas $(3,15$ y 3,10 , respectivamente). El aspecto peor valorado se obtuvo en las diferencias en el aprendizaje entre beneficiarios y no beneficiarios del programa $(2,80)$ (Figura 6).

\section{Figura 6}

Valoración de los Directores de Centros Escolares sobre el comportamiento de los indicadores educativos en el marco del programa Red de Oportunidades

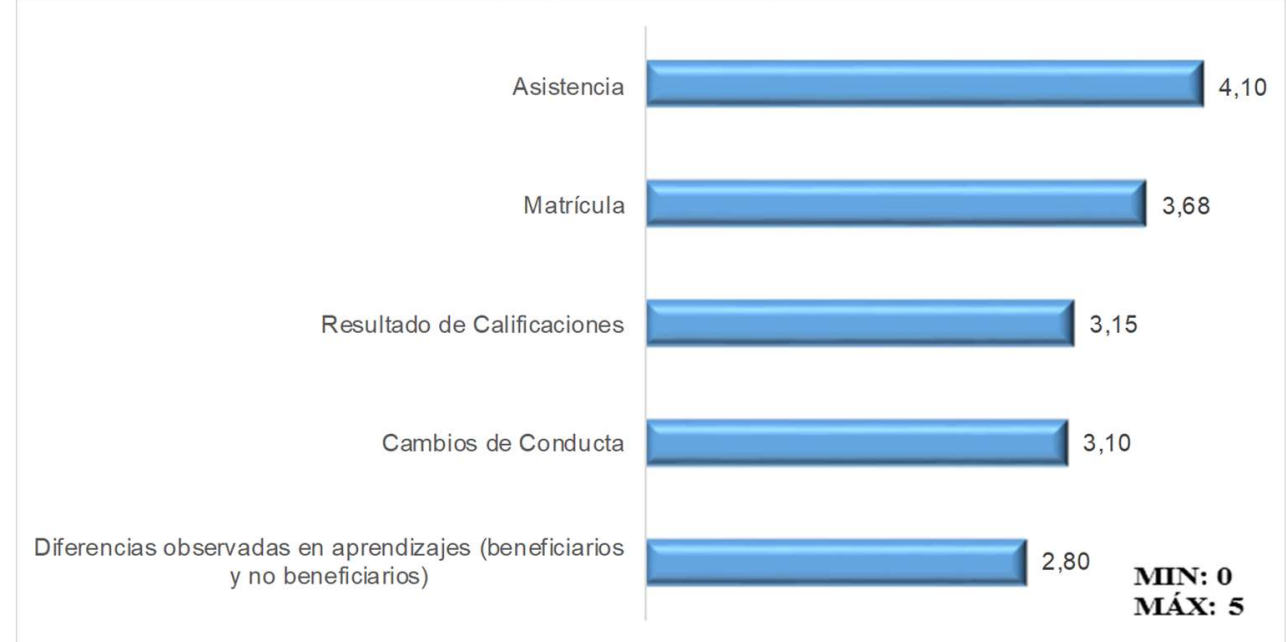

Fuente: Adaptado de Him (2017:127).

Sin embargo, empíricamente, no se ha demostrado que la condicionalidad en educación sea una condición suficiente para el incremento de la matrícula escolar, si bien parece poco probable que una transferencia de efectivo demasiado pequeña pueda inducir a los beneficiarios a ingresar en la escuela 
(Handa y Davis, 2006). A ello hay que añadir la necesidad de contar con las inversiones necesarias para mejorar las infraestructuras y la calidad de la oferta en las áreas y/o regiones participantes. Si estas son demasiado bajas, es difícil que puedan impulsar cambios significativos en el comportamiento de los beneficiarios. Además, la condicionalidad sobre la asistencia escolar podría no tener mucho sentido en países con ingresos medios, donde este indicador arroja resultados satisfactorios (Him, 2017). Por esta razón, una opción que podría plantearse es que las transferencias estuvieran condicionadas a la obtención de mejores resultados en el aprendizaje (Villatoro, 2008).

Estas valoraciones indican que el programa ha permitido mejorar indicadores como la matrícula y la asistencia escolar; sin embargo, no está claro si esos efectos positivos se deben a un mayor incremento del ingreso, producto de la transferencia monetaria que reciben las familias beneficiarias ("efecto ingreso"), o a los compromisos que deben adquirir para obtenerla ("efecto condicionalidad") (Cecchini, 2014).

El seguimiento de la corresponsabilidad en educación se ha realizado mediante el Sistema de Verificación de Corresponsabilidades (SISVCO). Durante el trabajo de campo se preguntó a los gestores del programa sobre el funcionamiento de esta herramienta. El 39\% de los entrevistados manifestó que es buena para capturar el control de matrícula de nuevos beneficiarios, porcentaje que se reducía hasta el $23 \%$ cuando se trata de llevar a cabo el seguimiento de la asistencia (Figura 7).

Figura 7

Valoración de los gestores sobre el funcionamiento del Sistema de Verificación de Corresponsabilidades (SISVCO)

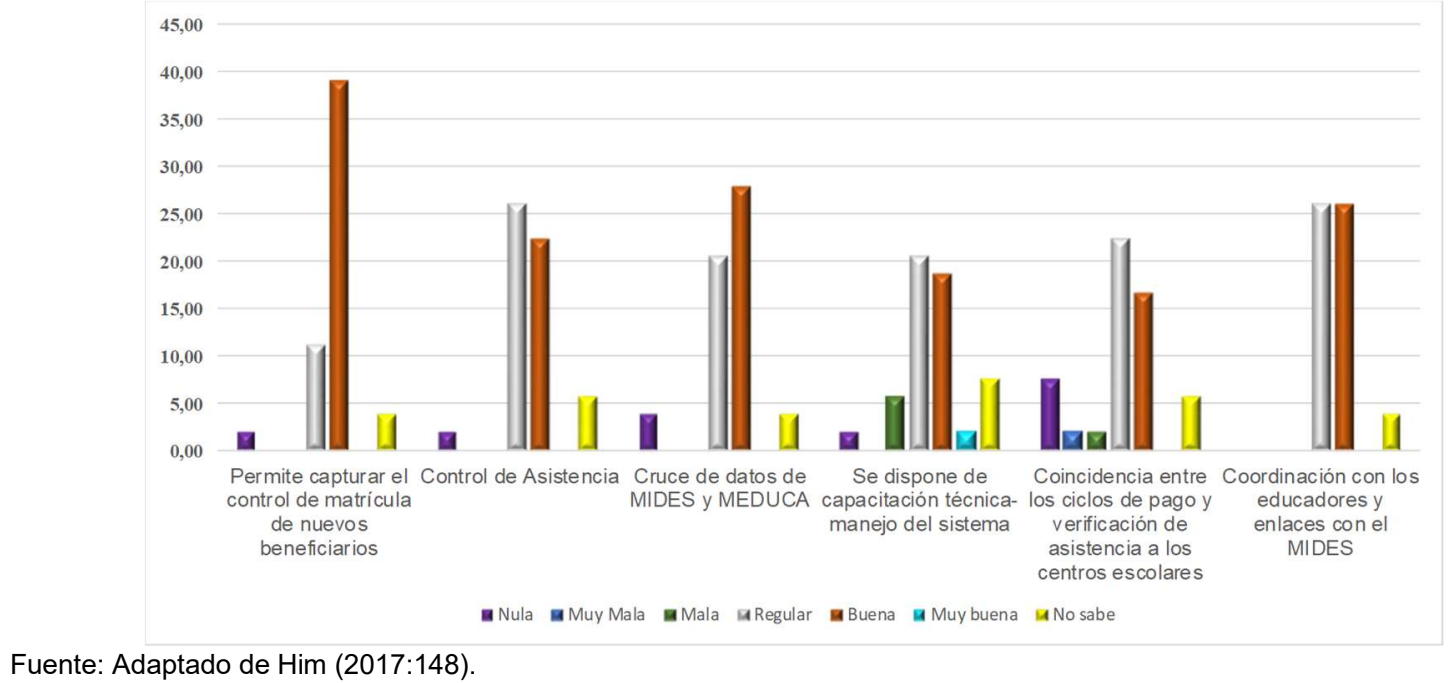

\subsection{Funcionamiento del sistema de seguimiento y evaluación}

Para que un sistema de seguimiento y evaluación funcione adecuadamente es esencial disponer de un buen sistema de indicadores. En términos generales, la regla básica descansa en utilizar un número reducido, que contengan información relevante y que estén claramente definidos para poder emitir juicios sólidos y conclusiones, con el fin de que puedan ser traducidos en mensajes claros dirigidos a los gestores públicos y a la sociedad en general. La importancia de los indicadores de seguimiento y evaluación reside en que posibilitan la medición de los efectos y avances de los programas públicos. Existe un vínculo entre la definición de los indicadores y la necesidad de diseñar métodos robustos que permitan medir el grado en que el cambio del indicador pueda ser atribuible a la intervención. Es responsabilidad del gestor público garantizar la disponibilidad de datos de calidad que provengan de diferentes fuentes de información (internas y externas).

El análisis del conjunto de indicadores del sistema de seguimiento y evaluación del programa Red de Oportunidades revela que han sido diseñados para informar sobre la evolución del programa y las actuaciones cofinanciadas por los organismos internacionales de crédito, probablemente por exigencia de estos últimos. Se dispone de 41 indicadores con valores anualizados y las metas a alcanzar, desde el año 2008 hasta 2014, indicando el medio por el que deben ser comunicados. Sin embargo, 16 de ellos, 
tal como aparecen definidos en la información proporcionada por el MIDES, no pueden considerarse indicadores en sentido estricto, más bien hacen referencia a la constitución de ciertas instituciones, mecanismos o procedimientos que, en todo caso, afectarían a la articulación institucional del programa $\mathrm{y}$, por ende, a su gobernanza, pero que nunca servirían para desempeñar las funciones de un indicador.

La Tabla 9 resume los resultados obtenidos en el trabajo de campo cuando los gestores fueron preguntados sobre las principales deficiencias del sistema de seguimiento y evaluación del programa Red de Oportunidades. Destaca como la más importante el retraso en la generación de información, que es valorada entre alta y muy alta por el 33,3\% de los entrevistados. En segundo lugar, se sitúan las deficiencias en el sistema de almacenamiento de la información, una limitación relevante para el 24,1\% de los encuestados. Por último, los problemas de los centros escolares para la verificación de las corresponsabilidades en educación es también una deficiencia preocupante para el $20,4 \%$ de los entrevistados.

Esta última valoración de los gestores se ve reforzada con el valor que alcanza el indicador que cuantifica el cumplimiento de la corresponsabilidad en educación, "porcentaje de niños de 4 a 17 años de edad que forman parte de la Red de Oportunidades que cumplan con la corresponsabilidad de educación (matrícula y asistencia) con relación al total de niños de la Red de Oportunidades en primaria y secundaria", según el cual sólo el $45 \%$ de los niños de primaria y secundaria beneficiarios de la Red de Oportunidades la cumplía. En contraposición, los indicadores definidos para medir el cumplimiento de la corresponsabilidad en salud son correctos y alcanzan valores bastante aceptables.

Tabla 9

Valoración de las deficiencias del sistema de seguimiento y evaluación del programa

\begin{tabular}{|c|c|c|c|c|c|c|}
\hline \multirow[b]{2}{*}{ Deficiencias identificadas } & \multicolumn{6}{|c|}{ Limitaciones (\%) } \\
\hline & Ninguna & Pocas & Moderadas & Altas & $\begin{array}{l}\text { Muy } \\
\text { altas }\end{array}$ & Ns/Nc \\
\hline Retraso en la generación de información & 14,8 & 16,7 & 22,2 & 25,9 & 7,4 & 13,0 \\
\hline Limitada disponibilidad de indicadores de realización & 7,4 & 5,6 & 50,0 & 9,3 & 9,3 & 18,5 \\
\hline $\begin{array}{l}\text { Deficiencias en el sistema de almacenamiento de } \\
\text { información }\end{array}$ & 16,7 & 14,8 & 27,8 & 24,1 & 0,0 & 16,7 \\
\hline $\begin{array}{l}\text { Problemas de coordinación en los centros escolares para } \\
\text { la verificación de las corresponsabilidades }\end{array}$ & 11,1 & 24,1 & 33,3 & 13,0 & 7,4 & 11,1 \\
\hline Falta de capacitación técnica & 11,1 & 7,4 & 53,7 & 7,4 & 1,8 & 18,5 \\
\hline Inadecuada coordinación institucional & 11,1 & 14,8 & 44,4 & 9,3 & 1,8 & 18,5 \\
\hline
\end{tabular}

Fuente: Adaptado de Him (2017:165).

Como ya se ha señalado, el sistema de seguimiento y evaluación de la Red de Oportunidades fue pactado al comienzo del programa con las organizaciones internacionales de crédito, al objeto de controlar el destino de los fondos, igual que el conjunto de indicadores, definiéndose los valores iniciales (valor actual y meta anual). Sin embargo, una de sus debilidades es la carencia de una línea base para algunos de ellos, hecho que limita las posibilidades de medición de los impactos atribuibles al programa. Tampoco están clasificados en función de la tipología que suele utilizarse para realizar el seguimiento y la evaluación de programas y políticas públicas (contexto, medios, realización, resultado e impacto), lo que impide construir la lógica de intervención y genera dificultades para realizar un buen seguimiento y posterior evaluación.

A su vez, la ausencia de encuestas longitudinales específicas, que permitan estudiar los cambios en las condiciones de vida de los beneficiarios, es otra de las limitaciones del sistema de seguimiento y evaluación del programa. Disponer de este tipo de datos hace posible identificar, cuantificar y caracterizar aquellos hogares beneficiarios del programa que lograron salir de su condición de pobreza o que mejoraron sus estándares de educación y salud. En definitiva, medir cambios socioeconómicos que ayuden a la toma de decisiones de los policy makers y orienten más eficientemente los gastos de la política social. Sin información cuantitativa de calidad es imposible medir los impactos atribuibles a un programa.

La insuficiencia del Sistema de Información Gerencial para sistematizar información relevante ha derivado en la creación de un sistema paralelo de cruce de información. Para Arraiz y Rozo (2011), en la práctica, el MIDES no ha desarrollado un sistema de información capaz de facilitar el seguimiento en el cumplimiento de las condiciones requeridas para las transferencias. 


\section{CONCLUSIONES}

Los programas de transferencias monetarias condicionadas llevan más de dos décadas aplicándose en numerosos países, siendo instrumentos muy controvertidos debido a que su implementación es compleja, al verse muy influenciados por los procesos políticos en los que se insertan. Además, estos programas requieren inversiones en infraestructuras y servicios (agua, educación, vivienda, salud, transporte, entre otros) para asegurar que el suministro sea capaz de responder, en calidad y cantidad suficientes, a la creciente demanda que se genera por el incentivo que supone la transferencia.

En el caso concreto del programa Red de Oportunidades, su implementación ha supuesto un paso notable a la hora de ofrecer un soporte a sectores vulnerables de la sociedad panameña. Sin embargo, este esfuerzo no ha sido suficiente. Es preciso incidir en aspectos de su gobernanza, como son: la focalización, para evitar los errores de inclusión y exclusión; el control del cumplimiento de la condicionalidad, que puede haber llevado a una relajación en las obligaciones comprometidas por parte de las familias beneficiarias, y que se ha mostrado como una de las principales debilidades del programa; la coordinación entre las instituciones involucradas en la intervención, sobre todo en el último eslabón de la cadena administrativa (entre los promotores sociales y los centros escolares, por un lado, y con los beneficiarios directos, por otro); una programación más participativa e incluyente; mejorar la formación de los gestores; y avanzar en mecanismos, procesos, registros, protocolos e indicadores que deriven en un sistema de seguimiento y evaluación adecuado para identificar las deficiencias, realizar las correcciones oportunas y generar información fiable y abierta a todos los sectores que permita rendir cuentas a la sociedad.

Entre las recomendaciones que cabría realizar, con carácter general, se han identificado dos. Por un lado, dada la existencia de errores de inclusión y exclusión, sería preciso contar con un criterio de focalización simple y transparente, así como con una definición objetiva y científica de los umbrales de elegibilidad; por otro, resulta crucial diseñar estrategias de graduación que permitan que los hogares beneficiarios no necesiten volver a utilizar el subsidio, para lo cual habría que redefinir la política con un enfoque que promueva cadenas productivas y áreas locales de empleo en las diferentes zonas de presencia del programa. Sin necesidad de ir tan lejos, y ciñéndonos a la condicionalidad en educación, podría plantearse la posibilidad de una transferencia variable (por ejemplo, según el número de hijos) y de mayor cuantía, que disminuyera de forma más significativa los costes de oportunidad de la educación de los hijos, así como reorientar el programa (y, en consecuencia, los recursos) hacia aquellos niveles educativos en los que aún se está lejos de la cobertura universal, e incluso que estuviera condicionada al logro de mejores resultados en el aprendizaje. Es deseable considerar el rendimiento cognitivo de los beneficiarios como indicador, máxime si se tiene en cuenta que podría tener efectos positivos a largo plazo para la mejora de la productividad y los salarios, con repercusiones favorables sobre la reducción de la pobreza.

\section{REFERENCIAS BIBLIOGRÁFICAS}

ARRAIZ, I. \& ROZO, S. (2011). Same Bureaucracy, Different Outcomes in Human Capital? How Indigenous and Rural Non-Indigenous Areas in Panama Responded to the CCT. Office of Evaluation and Oversight, IDB, Working Paper No. 03/11.

BARRETT, S. \& KIDD, S. (2015). The design and management of cash transfer programmes: an overview. KfW Development Bank (3), 1-21.

BARRIENTOS, A. \& DEJONG, J. (2006). Reducing Child Poverty with Cash Transfers: A Sure Thing? Overseas Development Institute (Eds.). Development Policy Review, 24(5), 537-552.

BARRIENTOS, A., GIDEON, J. \& MOLYNEUX, M. (2008). New developments in Latin America's social policy. Development and Change, 39(5), 759-774.

BETTS, J. AND WEDGWOOD, H. (2011). Effective institutions and good governance for development: Evidence on progress and the role of aid. Evaluation Insights, Number 4, 1-11. Recuperado de: https://www.oecd.org/derec/unitedkingdom/50313780.pdf

CECCHINI, S. (2014). Educación, Programas de Transferencias Condicionadas y protección social en América Latina y el Caribe. En: M. Feijoó y M. Poggi, Educación y Políticas Sociales. Sinergias para la inclusión. Buenos Aires: IIPE-UNESCO, 49-76.

CECCHINI, S. y ATUESTA, B. (2017). Programas de transferencias condicionadas en América Latina y el Caribe. Tendencias de cobertura e inversión. Santiago: Comisión Económica para América Latina y el Caribe. 
DE BRAUW, A. \& HODDINOTT, J. (2011). Must Conditional Cash Transfer Programs Be Conditioned to Be Effective? The Impact of Conditioning Transfers on School Enrollment in Mexico. Journal of Development Economics, 96(2), 359-370.

EUROPEAN COMMISSION (2012). Evalsed: The Resource for the evaluation of Socio-Economic Development. Bruselas: Unión Europea.

- (2014). Capturing the Success of your RDP: Ex post evaluation of 2007-2013 RDPS. Bruselas: Recuperado de: http://enrd.ec.europa.eu/evaluation

GÓMEZ HERMOSILLO, R. (2009). Evaluación Operativa de la Red de Oportunidades Panamá. Panamá: Ministerio de Desarrollo Social.

GRIN, J. \& LOEBER, A. (2007). Theories of Policy Learning: Agency, Structure and Change. En: F. Fischer, G.J. Miller y M.S. Sisney (Comps.). Handbook of Public Policy Analysis: Theory, Politics and Methods. New York: CRC Press, 201-219.

HANDA, S. \& DAVIS, B. (2006). The Experience of Conditional Cash Transfers in Latin America and the Caribbean. Development Policy Review, 24(5), 513-536. doi:10.1111/j.1467-7679.2006.00345.x

HIM CAMAÑO, M. (2017). Las transferencias monetarias condicionadas como instrumento de reducción de la pobreza: Incidencia sobre la formación de capital humano del Programa Red de Oportunidades (2010-2014) en Panamá. (Tesis Doctoral en Economía, no publicada). Universidad de Valladolid. Valladolid (España).

HIM CAMAÑO, M., OGANDO CANABAL, O. y MIRANDA ESCOLAR, B. (2016). Las transferencias monetarias condicionadas como instrumento de reducción de la pobreza: Articulación institucional del Programa Red de Oportunidades de Panamá (2010-2014). En: Aranda García, E. (Dir), Casares Ripol, J. y Cuadrado Roura, J. (Coord.): Política económica. Un contexto de crisis, recuperación e incertidumbre. Pamplona: Thomson Reuters-CIVITAS, 341-365.

INTERNATIONAL NGO TRAINING AND RESEARCH CENTRE (2013). Support to Civil Society: Emerging Evaluation Lessons. Evaluation Insights, Number 8, 1-14.

KIDD, S. \& WYLDE, E. (2011). Targeting the Poorest: An assesment of the proxy means test methodology. Canberra: Australian Government. AusAID.

LEGERO, J. (2011). Dos métodos de evaluación: Criterios y Teoría de Programas. Instituto Universitario de Estudios Europeos. CEU. Universidad de Sao Pablo. Documento de trabajo.15, 1-67.

LEHTONEN, M. (2005). OECD Environmental Performance Review Programme Accountability (f)or Learning? Evaluation, 11, 2: 169-188.

LEHTONEN, M. (2006). Deliberative Democracy, Participation, and OECD Peer Reviews of Environmental Policies. American Journal of Evaluation, 27, 2, Junio: 185-200

MINISTERIO DE DESARROLLO SOCIAL (2009). Manual Operativo Versión 5. Panamá: Red de Oportunidades. Protección Social.

MIRANDA ESCOLAR, B., ROJO GARCÍA J.L., GÓMEZ VALLE, I. y CAVERO ÁLVAREZ, J.C. (2015). El análisis contrafactual en la evaluación de políticas públicas: Una aproximación a través del estudio del caso. XII Jornadas Internacionales de Política Económica. Toledo, 28 y 29 de mayo.

MULLER, C. \& BIBI, S. (2010). Refining Targeting against Poverty Evidencie from Tunisia. Oxford Bulletin of Economics And Statistics, 73(3), 0305-9049.

PROGRAMA DE NACIONES UNIDAS PARA EL DESARROLLO (2015). Los tiempos de la polítización. Desarrollo Humano en Chile. Santiago de Chile.

ROGERS, P. (2014). La teoría del cambio. Síntesis metodológicas: evaluación de impacto, $n^{\circ} 2$. Florencia: Centro de Investigaciones de UNICEF.

VILLATORO, P. (2008). CCTs in Latin America: Human Capital Accumulation and Poverty Reduction. Poverty in Focus, 15, 14-15.

WATERS, W. (2009a). Diseño de Políticas y Programas Sociales. Estudio de Evaluación Cualitativa del programa Red de Oportunidades. Informe 1: Comarcas Indígenas.

WATERS, W. (2009b). Diseño de Políticas y Programas Sociales. Estudio de Evaluación Cualitativa del Programa Red de Oportunidades. Informe 2: Áreas Rurales y Urbanas. Versión 1.

WEISS, C.H. (1987). Evaluating Social Programs: What Have We Learned? Society 25(1), 40-45. 